\title{
INTEGRAL INEQUALITIES OF LEVINSON'S TYPE IN TIME SCALE SETTINGS
}

\author{
JosiPA BARIĆ, JOSIP PEČARIĆ AND DAJANA RADIŠIĆ
}

Abstract. A new class of functions, $\mathscr{K}_{1}^{c}(I)$, has been recently introduced by Baloch, Pečarić and Praljak. The authors proved that $\mathscr{K}_{1}^{c}(I)$ is the largest class of functions for which Levinson's inequality holds under Mercer's assumptions. We obtain Levinson's type inequalities in time scale settings by using the class $\mathscr{K}_{1}^{c}(I)$ and some known results regarding integral inequalites for convex (concave) functions on time scale sets.

Mathematics subject classification (2010): 26D15, 26A51.

Keywords and phrases: Levinson's inequality, Jensen's inequality, time scale calculus.

\section{REFERENCES}

[1] S. ABRAmovich, J. BARIĆ, J. PeČARIĆ, A variant of Jensen's inequality of Mercer's type for superquadratic functions, J. Inequal. Pure Appl. Math., 9, 3 (2008).

[2] R. P. Agarwal, M. Bohner, A. Peterson, Inequalities on time scale: a survey, Mathematical Inequalities and Applications, 4, 4, (2001), 535-557.

[3] M. ANWAR, R. BIBI, M. BOHNER, J. PEČARIĆ, Integral inequalities on time scale via the theory of isotonic linear functionals, Abstract and Applied Analysis, 2011, article id 483595, 16pp.

[4] I. A. BALOCH, J. PeČAriĆ, M. PraljaK, Generalization of Levinson's inequality, J. Math. Inequal., 9, 2 (2015), 571-586.

[5] J. BARIĆ, M. BOhner, R. JAKŠIĆ, J. PEČArić, Converses of Jessen's inequality on time scales, Mathematical Notes, 98, 1 (2015), 11-24.

[6] J. Barić, R. Bibi, M. Bohner, A. Nosheen, J. Pečarić, Jensen Inequalities on Time Scales, Theory and Applications, Zagreb, Element, 2015., monograph in inequalities.

[7] M. Bohner And G. SH. Guseinov, Multiple integration on time scales, Dynam. Systems Appl. 14 3-4, (2005) 579-606.

[8] M. Bohner AND G. SH. Guseinov, Multiple Lebesgue integration on time scales, Adv. Difference Equ., 2006, Art. ID 26391, 12 pp.

[9] M. Bohner, A.Peterson, Dynamic Equations on Time Scales, Birkhäuser, Boston, Basel, Berlin, 2001.

[10] P. S. Bullen, An inequality of N. Levinson, Univ. Beograd Publ. Elektrotehn. Fak. Ser. Mat. Fiz. 421-460 (1973), 109-112.

[11] W. S. Cheung, A. Matković, And J. PeČArić, A variant of Jessen's inequality and generalized means, J. Inequal. Pure Appl. Math., 7 1, (2006).

[12] S. Hilger, Ein Maßkettenkalkül mit Anwendung auf Zentrumsmannigfaltigkeiten, Phd. D. thesis, Universität Würzburg, 1988.

[13] S. Hilger, Analysis on measure chains - a unified approach to continuous and discrete calculus, Results Math. 18, 1-2 (1990), 18-56.

[14] S. HILGER, Differential and difference calculus unified, Nonlinear Anal. 30, 1 (1997), 143-166.

[15] B. Kaymakcalan, V. Lakshmikantham, S. Sivasundaram, Dynamic Systems on Measure Chains, Kluwer Academic Publishers, Dordrecht, 1996.

[16] N. Levinson, Generalization of an inequality of Ky Fan, J. Math. Anal. Appl. 8 (1964), 133-134.

[17] A. MCD. Mercer, A variant of Jensen's inequality, J. Inequal. Pure Appl. Math., 4 4, article 73, 2 pp. (electronic), 2003. 
[18] A. MCD. MERCER, Short proof of Jensen's and Levinson's inequalities, Math. Gazette 94 (2010), 492-495.

[19] J. PeČARIĆ, M. PrAlJAK, A. WitKows Ki, Generalized Levinson's inequality and exponential convexity, Opuscula Math. 35, 3 (2015), 397-410.

[20] T. Popoviciu, Sur une inealite de N. Levinson, Mathematica (Cluj) 6 (1964), 301-306.

[21] A. Witkowski, On Levinson's inequality, RGMIA Research Report Collection 15 (2012), Article 68.

[22] F.-H. Wong, C.-C. YeH, W.-C. LIAN, An extension of Jensen's inequality on time scales, Advances in Dynamical Systems and Applications, 1, 1, (2006), 113-120. 